\title{
Nosocomial Achromobacter xylosoxidans Infection Presenting as a Cavitary Lung Lesion in a Lung Cancer Patient
}

\author{
Vinoja Sebanayagam ${ }^{1}$, Paul Nguyen ${ }^{2}$, Mo'ath Nassar ${ }^{1}$, Ayman Soubani ${ }^{1}$ \\ 1. Pulmonary, Critical Care and Sleep Medicine, Wayne State University School of Medicine, Detroit, USA 2. Internal \\ Medicine, Wayne State University School of Medicine, Detroit, USA
}

Corresponding author: Vinoja Sebanayagam, vsebanayagam@gmail.com

\begin{abstract}
Achromobacter xylosoxidans is a Gram-negative bacillus that is known to cause nosocomial infections, primarily in patients with hematological malignancies. The most common primary manifestation is bacteremia. We report a novel case of primary A. xylosoxidans infection presenting as a cavitary lung lesion with associated pneumonia in a lung cancer patient who showed no evidence of malignant disease progression after radiation therapy. Our patient was initially admitted for acute hypoxic respiratory failure requiring mechanical ventilation. Initial computed tomography (CT) revealed a cavitary lesion in the right upper lobe of the lung. Diagnostic bronchoscopy with bronchoalveolar lavage (BAL) was performed and was negative for infectious etiologies including tuberculosis (TB) and fungal infections. Cytology was also negative for malignancy. However, the bacterial culture grew A. xylosoxidans. Antimicrobial therapy was initiated based on culture susceptibilities and the patient showed significant improvement in oxygen requirements. Due to poor functional status, the palliative care route was pursued and mechanical ventilation weaning was not performed. Cavitary pulmonary infections secondary to $A$. xylosoxidans are rarely reported in the medical literature. After conducting a thorough PubMed database search of the medical literature, we believe this is the first case of A. xylosoxidans infection manifesting as a cavitary lung lesion with associated pneumonia in a lung cancer patient.
\end{abstract}

Categories: Internal Medicine, Pulmonology

Keywords: achromobacter xylosoxidans, lung cavity, pneumonia

\section{Introduction}

Achromobacter xylosoxidans is an aerobic, non-fermenting Gram-negative bacillus that is capable of oxidizing xylose $[1,2]$. It is known to cause healthcare-associated infections, predominantly in immunocompromised individuals [1]. Within this group of individuals, it has been more commonly seen in patients with hematological malignancies [3-6]. The most common clinical presentation appears to be primary bacteremia, followed by pneumonia, and catheter-associated bacteremia [7]. Other reported presentations include meningitis, cellulitis, pyelonephritis, and endocarditis $[1,7,8]$.

Received 08/03/2020

Review began 08/04/2020 Review ended 08/08/2020 Published 08/17/2020

\section{(c) Copyright 2020}

Sebanayagam et al. This is an open access article distributed under the terms of the Creative Commons Attribution License CC-BY 4.0., which permits unrestricted use, distribution, and reproduction in any medium, provided the original author and source are credited.
Cavitary lung lesions are typically observed in a variety of diseases, including infections, malignancies, and autoimmune diseases [9]. The most commonly reported infectious etiologies include pulmonary tuberculosis (TB), common bacteria such as Staphylococcus Aureus, and fungi such as aspergillosis [9,10]. Cavitary lung lesions caused by A. xylosoxidans are extremely rare in the medical literature [11]. The case report we found in the literature was a middle-aged immunocompetent man who had a community-acquired $A$. xylosoxidans induced cavitary lung lesion that responded to antibiotics [11]. However, to the best of our knowledge, there are no reports in the literature of $A$. xylosoxidans associated cavitary lung lesions in lung cancer patients. Therefore, we report a novel case of $A$. xylosoxidans manifesting as a cavitary lung lesion in a lung cancer patient who did not show signs of cancer progression after radiation therapy.

\section{Case Presentation}

A 69-year-old woman with a past medical history of chronic obstructive pulmonary disease and stage IA2 squamous cell carcinoma of the right upper lobe of the lung was diagnosed with the malignancy one year prior to the current hospital admission. The patient was treated with stereotactic body radiation therapy (SBRT) to the right upper lobe, due to poor performance status and advanced chronic obstructive pulmonary disease. Computed tomography of the chest (CT-thorax) performed three months prior to the current admission showed significant improvement in the size of the malignant lesion (Figure 1). In the current admission, the patient presented to the hospital with shortness of breath and was admitted to the intensive care unit (ICU) for the management of acute hypoxic respiratory failure requiring invasive mechanical ventilation. Initial diagnostic workup including complete blood count, basic metabolic panel, and microbiological studies was unremarkable. 


\section{Cureus}

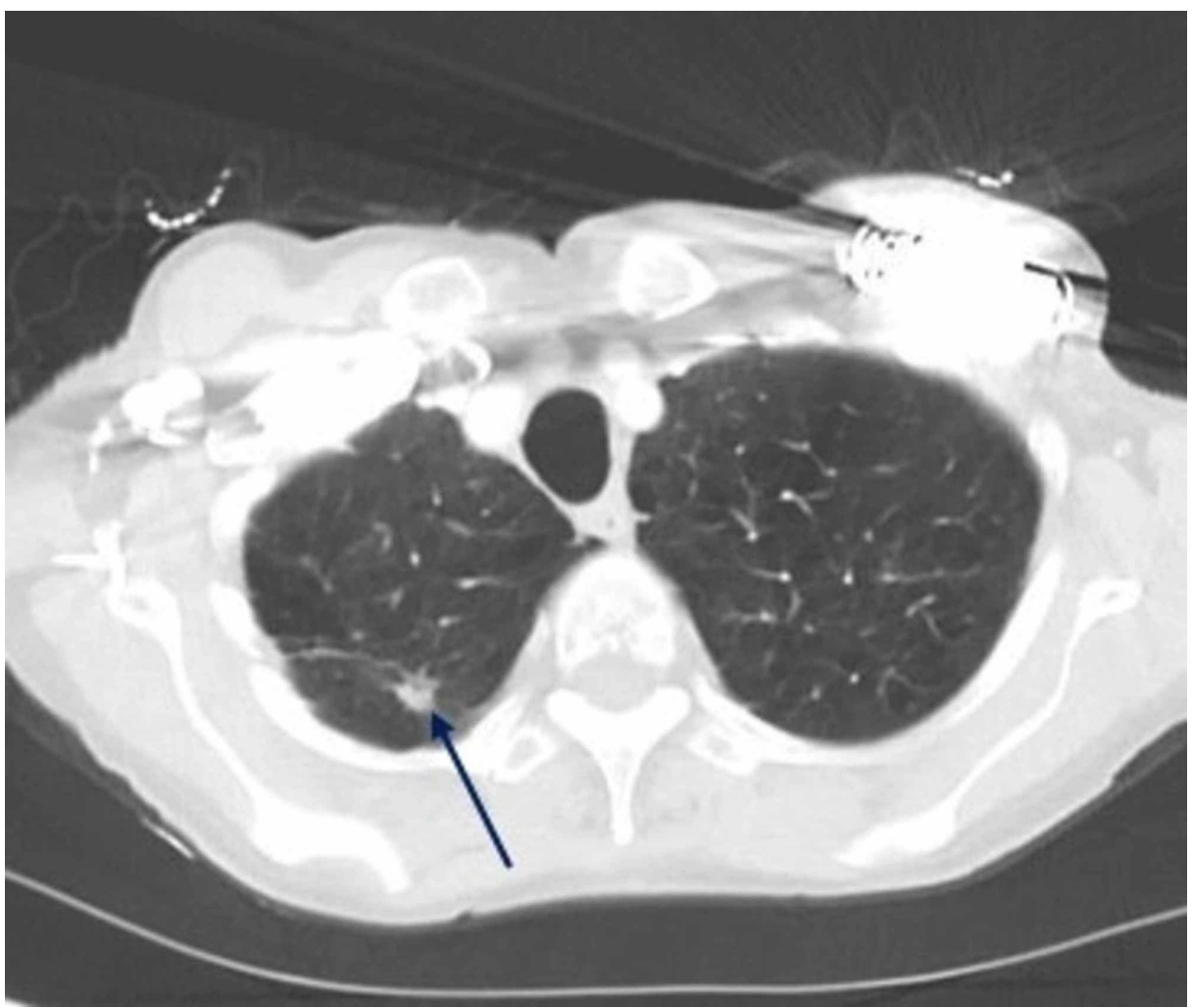

FIGURE 1: CT-thorax showing a right upper lobe malignant lung nodule (blue arrow), after radiation therapy

A CT-thorax revealed extensive airspace consolidation in the right upper lobe of the lung with a $3.8 \mathrm{~cm}$ gasfilled irregular cavity surrounded by consolidation (Figure 2). Broad spectrum antimicrobial therapy was initiated and included imipenem.

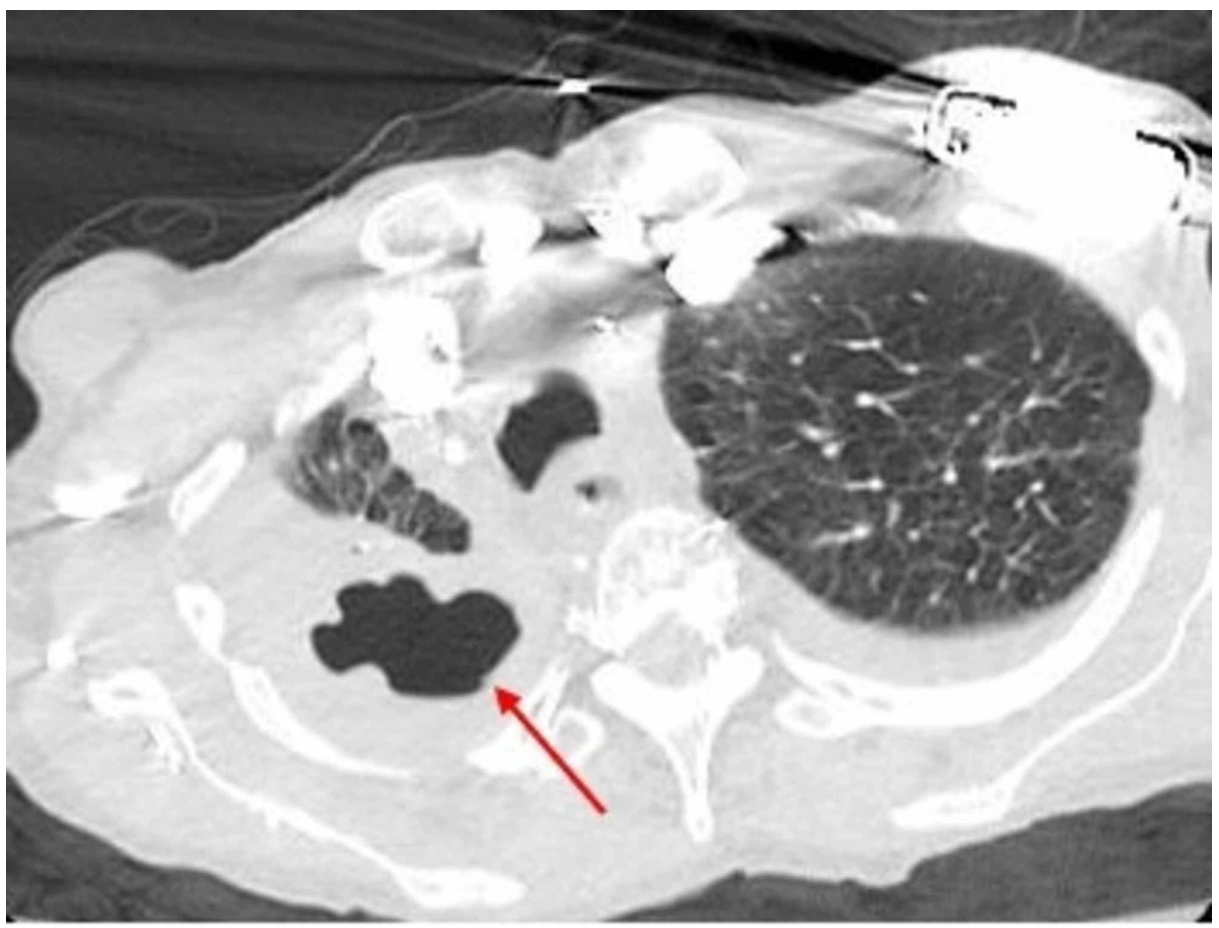

FIGURE 2: CT-thorax obtained at admission showing a gas-filled cavitary lesion (red arrow) surrounded by consolidation, in the upper lobe of the right lung 


\section{Cureus}

Diagnostic bronchoscopy with bronchoalveolar lavage (BAL) was performed and resulted in the growth of $A$. xylosoxidans on microbiological culture (Table 1 shows antibiotic sensitivity testing). The BAL was negative for TB or fungal infections. The cytological analysis revealed no evidence of malignant cells.

\begin{tabular}{lll}
\hline Microorganism: A. xylosoxidans in the BAL & MIC $(\mu \mathrm{g} / \mathrm{mL})$ & Interpretation \\
Antibiotic & $>32$ & $\mathrm{R}$ \\
Amikacin & $>16$ & $\mathrm{R}$ \\
Aztreonam & 16 & $\mathrm{MS}$ \\
Cefepime & 8 & $\mathrm{~S}$ \\
Ceftazidime & $>32$ & $\mathrm{R}$ \\
Ceftriaxone & $>2$ & $\mathrm{R}$ \\
Ciprofloxacin & $>8$ & $\mathrm{R}$ \\
Gentamicin & 2 & $\mathrm{~S}$ \\
Imipenem & $\leq 2 / 4$ & $\mathrm{~S}$ \\
Piperacillin/Tazobactam & $>8$ & $\mathrm{R}$ \\
Tobramycin & $\leq 0.5 / 9.5$ & $\mathrm{~S}$ \\
Trimethoprim/Sulfamethoxazole & &
\end{tabular}

\section{TABLE 1: Antibiotic sensitivity results}

BAL: bronchoalveolar lavage, MIC: minimum inhibitory concentration; S: sensitive; R: resistant; MS: moderately sensitive.

CT-thorax was repeated one week after initiation of treatment and showed stable cavitary lesion (Figure 3). Oxygen requirement improved significantly and weaning of mechanical ventilation was achieved. However, re-intubation was performed due to excessive secretions and inability to protect airways. After goals of care discussion, the patient and family decided to pursue palliative care.

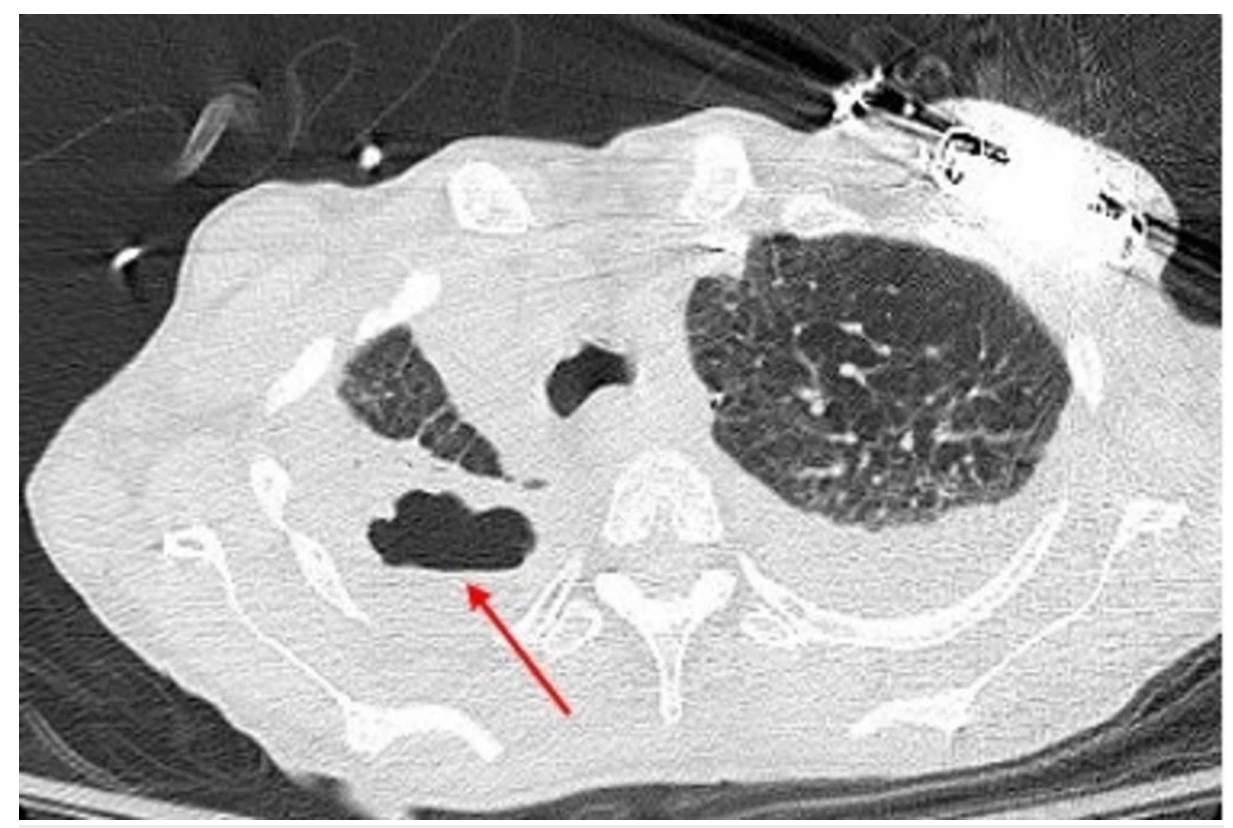

FIGURE 3: CT-thorax obtained one week after treatment initiation showing stable right upper lobe cavitary lesion (red arrow) with 


\section{Cureus}

\section{surrounding consolidation}

A repeat sputum culture obtained 2.5 weeks after the bronchoscopy, continued to be positive for $A$. xylosoxidans susceptible to imipenem (Figure 4 is an image of the Gram stain from our patient).

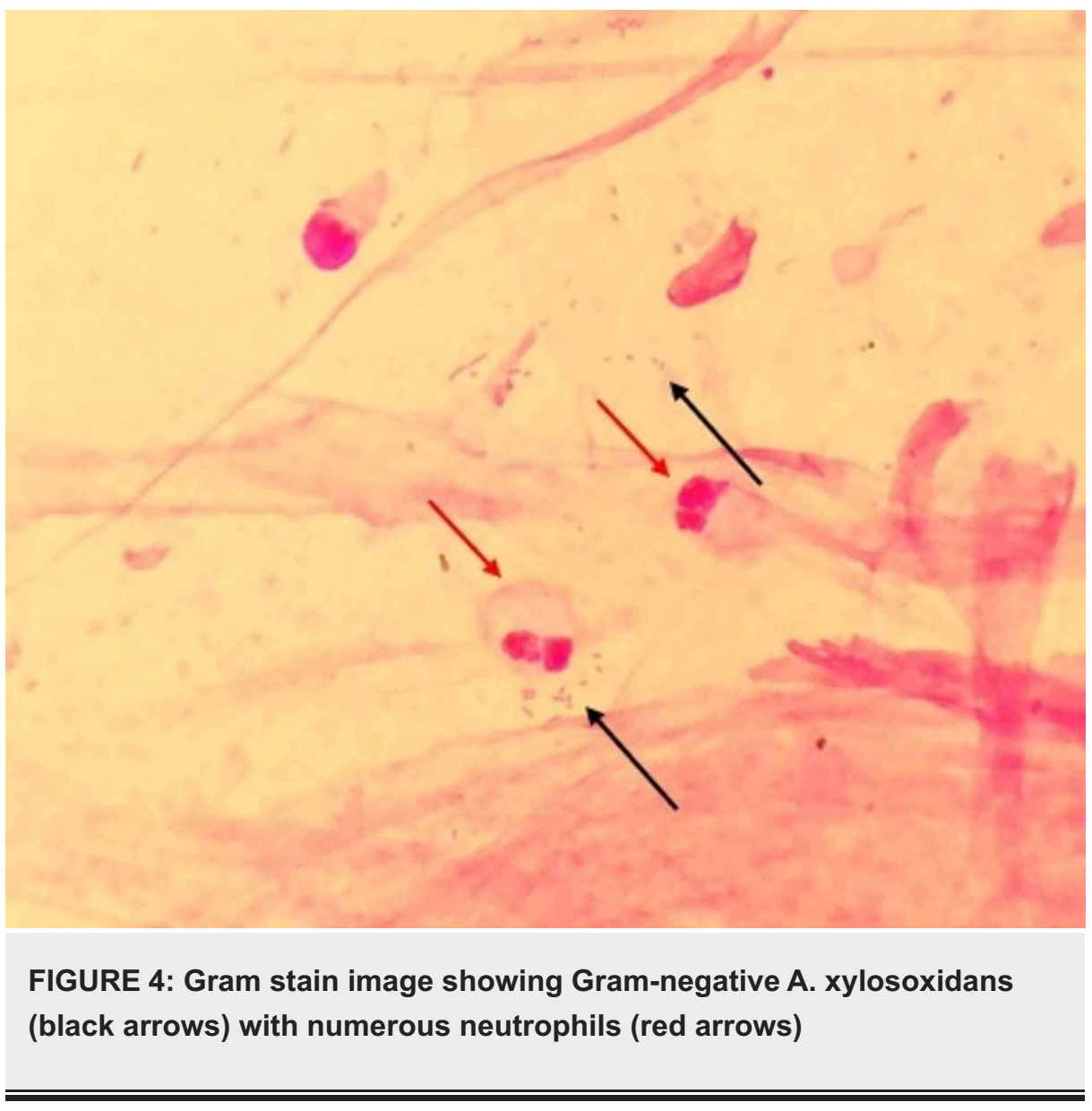

\section{Discussion}

A. xylosoxidans was first described by Yabuchi et al. after it was isolated from the ear discharge of patients with chronic otitis media [2]. It is widely present in aqueous environments including well water and swimming pools [12]. However, it is also known to be a nosocomial colonizer that has been isolated from solutions found in the healthcare setting, including dialysis solutions, and chlorhexidine gluconate solutions, as well as fomites such as mechanical ventilators, intravenous catheters, and urinary catheters [12]. A. xylosoxidans is an opportunistic pathogen that primarily affects patients with underlying medical issues such as malignancy (30\%), cardiac disease (21\%), and immunosuppression (27\%) [12]. It primarily presents as uncomplicated bacteremia, but has also caused pneumonia, soft tissue infections, and urinary tract infections among others, and has been isolated from multiple sources including blood, cerebrospinal fluid, sputum, skin, etc. [12]. We were able to find only one case during our literature search, in which this bacteria presented as a cavitary lung lesion [11]. However, this presentation is different from our case in that it stemmed from a community-acquired infection in an otherwise healthy immunocompetent man who had no significant past medical history or recent hospitalizations [11].

The two main differential diagnoses for cavitary lung lesions in adults are malignant tumors and benign infections [9]. Our patient has a history of squamous cell cancer, presenting as a right upper lobe nodule which subsequently decreased in size after radiation therapy. Figure 1 shows the CT-thorax performed three months prior to the current hospital admission, demonstrating the right upper lobe malignant nodule. Bronchial wash cytologic analysis from the BAL was negative for malignant cells. While it has low sensitivity for cancer diagnosis [13], it is unlikely that the malignancy had progressed significantly over the course of three months. This makes infectious etiology more likely especially in the light of positive BAL culture. Just three days prior to the current admission, our patient had been discharged after being admitted and treated for acute encephalopathy secondary to a urinary tract infection. Given that A.xylosoxidans is a wellestablished nosocomial colonizer, it is very likely that the bacterial inoculation occurred during her recent hospital stay. Furthermore, significant improvement in oxygen requirement was noted upon treatment with appropriate antimicrobial therapy during the current admission. 
From a treatment standpoint, consistent with previous antibiotic susceptibility profiles reported in the literature [1], A. xylosoxidans isolated from our BAL sample was susceptible to imipenem. Based on in vitro susceptibility tests reported in the literature, empirical antibiotic coverage that includes a carbapenem agent is usually adequate to treat A. xylosoxidans infections [1]. Imipenem is known to have adequate lung penetration [14]. Overall, our patient received empiric imipenem coverage for a total of eight days with an improvement in oxygen requirements. However, no significant improvement in the size of the cavitary lesion was noted on the imaging done a week after initiation of treatment. Optimally, significant changes in chest imaging are noted six to eight weeks after completion of therapy; however, this was not appropriate for our patient as she pursued palliative care. Of note, the repeat sputum culture obtained 2.5 weeks after the bronchoscopy was positive for $A$. xylosoxidans, reinforcing the notion that the bacterium served as a primary causative agent of infection rather than just a contaminant. The persistence of the bacterium in the respiratory culture could be attributed to the inadequate duration of antibiotic coverage, as the bacterium isolated from the sputum culture was still susceptible to imipenem.

\section{Conclusions}

In this case report, we present a case of A. xylosoxidans infection that manifested as a single cavitary lung lesion in the setting of pneumonia, in a lung cancer patient who showed no radiographical signs of cancer progression after radiation therapy. The novelty of this case lies in the way this bacterium presented in our patient. We present the first case of a nosocomial $A$. xylosoxidans infection manifesting as a single cavitary pulmonary lesion in a lung cancer patient.

\section{Additional Information \\ Disclosures}

Human subjects: Consent was obtained by all participants in this study. Conflicts of interest: In compliance with the ICMJE uniform disclosure form, all authors declare the following: Payment/services info: All authors have declared that no financial support was received from any organization for the submitted work. Financial relationships: All authors have declared that they have no financial relationships at present or within the previous three years with any organizations that might have an interest in the submitted work. Other relationships: All authors have declared that there are no other relationships or activities that could appear to have influenced the submitted work.

\section{References}

1. Barragán EP, Pérez JS, Corbella L, Orellana MÁ, Fernández-Ruiz M: Achromobacter xylosoxidans bacteremia: clinical and microbiological features in a 10-year case series. Rev Esp Quimioter. 2018, 31:268273.

2. Yauuuchi E, Yano I, Goto S, Tanjmura E, Ito T, Ohyama A: Description of Achromobacter xylosoxidans Yabuuchi and Ohyama 1971. Int J Syst Bacteriol. 1974, 24:470-477. 10.1099/00207713-24-4-470

3. Tugcu D, Turel O, Aydogan G, et al.: Successful treatment of multiresistant Achromobacter xylosoxidans bacteremia in a child with acute myeloid leukemia. Ann Saudi Med. 2015, 35:168-169. 10.5144/02564947.2015.168

4. Hernandez J, Martino R, Pericas R, Sureda A, Brunet S, Domingo-Albos A: Achromobacter xylosoxidans bacteremia in patients with hematologic malignancies. Haematologica. 1998, 83:284-285.

5. Dai J, Huen AO, Kestenbaum LA, Sarezky MD, Coughlin CC, Yan AC: Achromobacter xylosoxidans bacteremia and cellulitis: a report of a case. Pediatr Dermatol. 2015, 32:186-187. 10.1111/pde.12608

6. Sammassimo S, Pruneri G, Andreola G, et al.: A retrospective international study on primary extranodal marginal zone lymphoma of the lung (BALT lymphoma) on behalf of International Extranodal Lymphoma Study Group (IELSG). Hematol Oncol. 2016, 34:177-183. 10.1002/hon.2243

7. Ramos JM, Domine M, Ponte MC, Soriano F: Bacteremia caused by Alcaligenes (Achromobacter) xylosoxidans: description of 3 cases and review of the literature. Enferm Infecc Microbiol Clin. 1996, 14:436-440.

8. Kikuchi T, Mori T, Kohashi S, et al.: Cellulitis due to Achromobacter xylosoxidans during bortezomib therapy for multiple myeloma. Rinsho Ketsueki. 2016, 57:175-179. 10.11406/rinketsu.57.175

9. Parkar AP, Kandiah P: Differential diagnosis of cavitary lung lesions . J Belgian Soc Radiol. 2016, 100:1-8. 10.5334/jbr-btr.1202

10. Gadkowski LB, Stout JE: Cavitary pulmonary disease. Clin Microbiol Rev. 2008, 21:305-333. 10.1128/CMR.00060-07

11. Hwang CH, Kim WJ, Jwa HY, Song SH: Community-acquired Achromobacter xylosoxidans infection presenting as a cavitary lung disease in an immunocompetent patient. Yeungnam Univ J Med. 2020, 37:5458. 10.12701/yujm.2019.00276

12. Claassen SL, Reese JM, Mysliwiec V, Mahlen SD: Achromobacter xylosoxidans infection presenting as a pulmonary nodule mimicking cancer. J Clin Microbiol. 2011, 49:2751-2754. 10.1128/JCM.02571-10

13. Rao S, Rao S, Lal A, Barathi G, Dhanasekar T, Duvuru P: Bronchial wash cytology: a study on morphology and morphometry. J Cytol. 2014, 31:63-67. 10.4103/0970-9371.138664

14. Van Hasselt JGC, Rizk ML, Lala M, et al.: Pooled population pharmacokinetic model of imipenem in plasma and the lung epithelial lining fluid. Br J Clin Pharmacol. 2016, 81:1113-1123. 10.1111/bcp.12901 